\title{
Reabilitação com prótese oculopalpebral após exenteração de órbita: relato de caso
}

Rehabilitation with oculopalpebral prosthesis after orbit exenteration: a case report

Rehabilitación con prótesis oculopalpebral después de la exenteración de la órbita: reporte de un caso

\author{
Ana Carolina dos Santos MENEZES ${ }^{1}$ \\ Luciana Ferreira STAHEL-LAGE ${ }^{2}$ \\ Lísia Daltro Borges ALVES ${ }^{1}$ \\ Fernanda Vieira HEIMLICH ${ }^{3}$ \\ Mônica Eliana Ribeiro MATOS ${ }^{4}$ \\ José Roberto de Menezes PONTES ${ }^{5}$ \\ Héliton Spíndola ANTUNES
}

${ }^{1}$ Cirurgiã dentista, Mestranda no programa de Oncologia do Instituto Nacional de Câncer José Alencar (INCA), Brasil

${ }^{2}$ Cirurgiã dentista, seção de Estomato Odontologia e Prótese, Instituto Nacional de Câncer José Alencar (INCA), Brasil

${ }^{3}$ Cirurgiã dentista, Residência Multiprofissional em Oncologia e Física Médica do Instituto Nacional de Câncer José Alencar (INCA),Brasil

${ }^{4}$ Técnica em Prótese Dentária, seção de Estomato Odontologia e Prótese, Instituto Nacional de Câncer José Alencar (INCA), Brasil

${ }^{5}$ Doutor em Odontologia, seção de Estomato Odontologia e Prótese, Instituto Nacional de Câncer José Alencar (INCA),Brasil

${ }^{6}$ Doutor em Oncologia, divisão de Pesquisa Clínica e Desenvolvimento Tecnológico do Instituto Nacional de Câncer José Alencar (INCA), Brasil

\section{Resumo}

Introdução: A ressecção cirúrgica de tumores em região de cabeça e pescoço é um tratamento eficaz, mas que implica em significativa desfiguração facial dependendo da localização da lesão. Aqueles pacientes considerados curados precisam ser reabilitados estética e funcionalmente para que possam ser reintegrados às funções sociais. A prótese maxilofacial é um artefato de baixo custo, que pode resolver essa necessidade. Relato de caso: Esse artigo relata o caso de uma paciente de 19 anos submetida à exenteração de órbita para tratamento de Tumor Fibroso Solitário Retrorbitário que, após a cirurgia e radioterapia adjuvante, foi reabilitada por meio de prótese óculopalpebral em silicone. Conclusão: O caso foi considerado um sucesso e ressalta a importância do cirurgião dentista na equipe oncológica e o positivo impacto psicológico e social da reabilitação.

Descritores: Evisceração do Olho; Exenteração Orbitária; Olho Artificial; Reabilitação; Sobrevivência; Ajustamento Social.

\section{Abstract}

Introduction: Surgical resection of head and neck tumors is an effective treatment, but it implies significant facial disfigurement depending on the location of the lesion. Those patients considered cured need to be rehabilitated aesthetically and functionally so that they can be reintegrated into social functions. The maxillofacial prosthesis is an artifact of low cost, which can solve this need. Case Report: This article reports the case of a 19-year-old patient who underwent orbit exanteration for the treatment of Solitary Retrorbital Fibrous Tumor, which, after surgery and adjuvant radiotherapy, was rehabilitated using an oculopebral prosthesis made of silicone. Conclusion: The case was considered a success and highlights the importance of the dental surgeon in the oncology team and the positive psychological and social impact of rehabilitation

Descriptors: Eye Evisceration; Orbit Evisceration; Eye, Artificial; Rehabilitation; Survival; Social Adjustment.

\section{Resumen}

Introducción: La resección quirúrgica de los tumores de cabeza y cuello es un tratamiento eficaz, pero implica una desfiguración facial significativa según la ubicación de la lesión. Aquellos pacientes considerados curados necesitan ser rehabilitados estética y funcionalmente para que puedan reintegrarse a las funciones sociales. La prótesis maxilofacial es un artefacto de bajo costo que puede resolver esta necesidad. Reporte del caso: Este artículo informa el caso de una paciente de 19 años que se sometió a una exenteración de órbita para el tratamiento del tumor fibroso retrorbital solitario, que, después de la cirugía y la radioterapia adyuvante, fue rehabilitada con una prótesis oculopalpebral de silicona. Conclusión: El caso se consideró un éxito y destaca la importancia del cirujano dental en el equipo de oncología y el impacto psicológico y social positivo de la rehabilitación.

Descriptores: Evisceración del Ojo; Evisceración Orbitaria; Ojo Artificial; Rehabilitación; Supervivencia; Ajuste Social.

INTRODUÇÃO

O câncer é uma das doenças crônicas de maior complexidade no cenário mundial atual. Segundo os dados do relatório de 2018 da Globocan/IARC, foram diagnosticados 17.036.901 casos de neoplasias, excluindo-se os cânceres de pele não melanoma ${ }^{1}$.

As neoplasias que afetam a região de cabeça e pescoço são um grupo heterogêneo $o^{2,3}$ e podem ter origem tanto epitelial, mesenquimal como também neural ${ }^{2}$. Afetam diversos sítios, dentre eles a cavidade oral, a pele, a orofaringe, a nasofaringe, a hipofaringe, a laringe, os seios paranasais, órbita e as glândulas salivares ${ }^{2,3}$.

Em casos nos quais a neoplasia se desenvolve na região orbital ou periorbital, a exenteração da órbita é geralmente 0 procedimento de eleição para tratamento ${ }^{4}$ e, dependendo do estadiamento do tumor, a adjuvância com quimioterapia ou radioterapia, isoladas ou combinadas, pode ser indicada ${ }^{5,6}$.
O tratamento cirúrgico, apesar de efetivo, implica em considerável desfiguração facial, o que pode acarretar uma série de problemas psicológicos e sociais para os pacientes $^{4,5,7-12}$. Esses são susceptíveis a quadros de depressão, ansiedade, fobia social, passividade, revolta e baixa autoestima ${ }^{9,12}$. A equipe de saúde, na qual o Cirurgião Dentista está inserido, deve ser treinada para auxiliar o paciente e seus familiares no preparo para o pós-operatório, procurando oferecer ajuda psicológica e reabilitação através de cirurgias e/ou próteses ${ }^{4,12}$. Desse modo, é possível proporcionar estética, função e reintegração do paciente ao meio social e familiar ${ }^{8,12-15}$. CASO CLÍNICO

Paciente do sexo feminino, 19 anos, matriculada no Instituto Nacional de Câncer (INCA) em março de 2018 com diagnóstico inicial de Linfangioma de órbita em olho direito. 
Negava a existência de outras comorbidades, assim como também negava tabagismo e etilismo. Em relação a alergias, informava somente reação à Amoxicilina. Relatava evolução de cinco anos desde a primeira manifestação ocular e alegava ter sofrido duas intervenções cirúrgicas nesse intervalo de tempo: a primeira, em 2015, na qual a lesão foi completamente excisada pela primeira vez, com diagnóstico histopatológico de Hemangioma Capilar; e a segunda, em 2016, após recidiva, com novo procedimento de excisão completa da lesão, dessa vez com diagnóstico histopatológico de Linfangioma.

Em 2018, a paciente procurou novamente atendimento com queixa de dor e cegueira no olho direito, informando que havia tido também quadros de sangramento e eliminação de secreção purulenta, além de febre. Após diagnóstico de infecção, foi prescrito ciprofloxacino e corticoides, com regressão da infecção e da sintomatologia. Ademais, realizouse ressonância magnética, na qual foi identificada imagem sugestiva de Malformação Vasculo-Linfática ou Linfangioma.

Na primeira avaliação no INCA, em maio de 2018, a paciente queixava-se de fortes dores e cegueira em olho direito. Ao exame clínico, a exoftalmia era evidente. Era possível também observar uma lesão intraorbitária medindo $7 \times 6 \times 4 \mathrm{~cm}$, com presença de edema e deslocamento do globo ocular - que mantinha boa mobilidade, apesar da perda da acuidade visual. O exame das cadeias linfonodais cervicais revelou ausência de linfonodos afetados. Foi solicitada a revisão de lâminas e a proposta de tratamento para o caso foi de exenteração de órbita, com fechamento primário do sítio cirúrgico. O procedimento foi realizado em maio de 2018, sem intercorrências.

$\mathrm{Na}$ análise histopatológica da peça cirúrgica constatou-se que a lesão consistia, na verdade, em um Tumor Fibroso Solitário Retrorbitário. Desse modo, a proposta de tratamento foi revista: a Seção de Cabeça e Pescoço indicou a radioterapia adjuvante que foi realizada na região da exenteração, com 64 Gy. Após consulta de controle na Seção de Cabeça e Pescoço, a doença foi considerada controlada e nessa data, foi solicitada avaliação da Seção de Odontologia para a confecção de prótese oculopalpebral visando reabilitação da paciente.

$\mathrm{Na}$ primeira consulta odontológica, em dezembro de 2019, a paciente se apresentou fazendo uso de tampão e, abaixo dele, era possível observar extensa reação alérgica em pele, possivelmente ao adesivo do tampão (Figura 1). A paciente informava estar fazendo uso desse desde a cirurgia por vergonha de a verem sem ele, já que trabalhava diretamente com o público e podia causar estranhamento devido a sua atual condição; era possível ver a frustração da paciente diante a situação e seu embaraço pela deformidade cirúrgica. Nessa primeira consulta, realizou-se a moldagem da cavidade para dar início ao processo de confecção da prótese. $\mathrm{Na}$ consulta subsequente, em janeiro de 2020 , realizou-se a prova em cera a estrutura e os ajustes necessários para a confecção da prótese final. Foram definidos nesse atendimento a cor da íris, o posicionamento da mesma no globo ocular que estava sendo confeccionado e foram realizados ajustes nas bordas da peça visando melhor encaixe na cavidade. A instalação da prótese se deu dois meses após o primeiro atendimento no setor. Consistia em uma prótese confeccionada em silicone, que deveria ser fixada ao rosto por meio de um adesivo apropriado (Figura 2). Nessa consulta, ao se observar pela primeira vez no espelho com a prótese em posição (Figura 3), foi possível observar a mudança na autopercepção da paciente em relação a sua condição, não somente pelos seus efusivos agradecimentos à equipe, mas também pela mudança no seu aspecto facial.

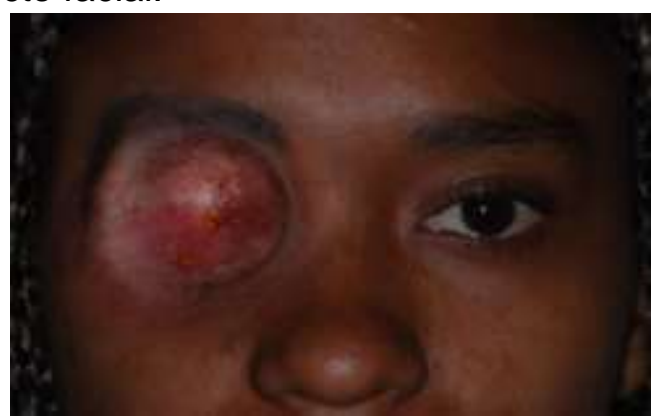

Figura 1: Cavidade resultante da cirurgia de exoftalmia. A foto foi tirada no segundo atendimento no ambulatório de Odontologia.

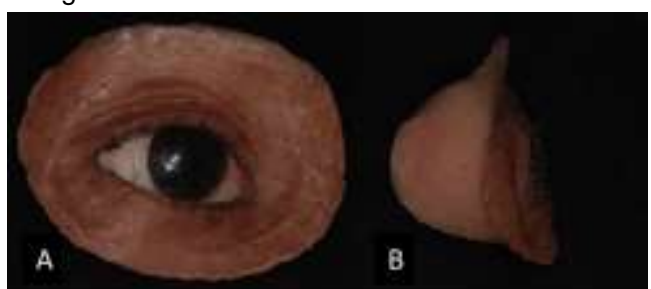

Figura 2: Prótese óculopalpebral; A. Vista frontal; B. Vista lateral.

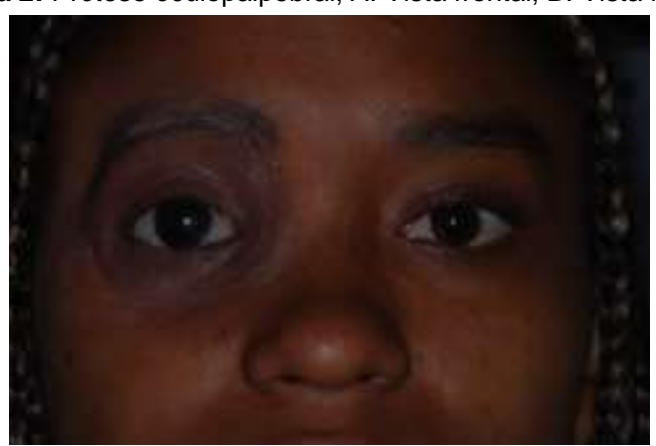

Figura 3: Paciente com a prótese oculopalpebral em posição. 
DISCUSSÃO

A sociedade contemporânea entende a face como um dos aspectos mais significativos na determinação da identidade e na integração social $^{4}$. Ademais, as cobranças impostas por essa sociedade em relação aos padrões de senso estético aceitável são cada vez mais reforçadas considerando a dominação exercida pela mídia e pelas redes sociais.

É lógico, portanto, concluir que pacientes com desfigurações faciais tendem a receber respostas negativas no convívio, tanto por exclusão social como por estigmatização; essas implicam em perda da habilidade de interação, que pode gerar problemas racionados a abusos físicos e verbais, comportamento hostil e isolamento ${ }^{4,5,7,11,12,16}$.

Pacientes candidatos à exenteração e também suas famílias necessitam ser orientados quanto às possíveis implicações psicológicas do procedimento antes que o mesmo ocorra ${ }^{4,5,14}$, já que a desconstrução da harmonia facial, mesmo que temporária, implica em trauma psicológico e desestruturação devido ao comprometimento do aspecto de normalidade, da harmonia e da simetria facial, além do comprometimento funcional ${ }^{4,5,7,8-12}$. Do mesmo modo, a equipe de saúde responsável por esse paciente deve não somente buscar a cura da patologia, mas o bem estar psicossocial desse paciente e a sua reabilitação à sociedade após o tratamento ${ }^{4,5,17}$.

Devemos compreender, desse modo, a importância de uma reabilitação como a que foi feita. Uma paciente jovem, tratada com sucesso para sua patologia e com uma expectativa de vida estendida pôde, por meio de uma prótese, de baixo custo, confeccionada em um curto espaço de tempo se enxergar novamente como um ser humano inteiro e pertencente a sociedade.

A prótese bucomaxilofacial é uma especialidade na Odontologia que busca reabilitar pacientes com grandes perdas de estrutura nas regiões de boca e face ${ }^{7}$, além de proteger os tecidos da área danificada ${ }^{14}$. Ela representa o primeiro passo na recuperação da qualidade de vida e do bem estar do paciente com deformidades faciais ${ }^{9,13,15,18}$, permitindo sua recuperação estética e funcional, além da reintegração ao meio social e familiar ${ }^{12,15,17 .}$

Esses dispositivos apresentam como vantagem a possibilidade de observação da cicatrização da ferida, a avaliação de recorrência de doença, a simplicidade técnica, o custo reduzido e, em determinadas situações, a superioridade estética?
Essas próteses podem ser de diferentes tipos, de acordo com a área que deverá ser reabilitada, podendo ser: oculares, nasais, oculopalpebrais (que foi o tipo usado para reabilitar essa paciente), auriculares, maxilofaciais, obturadoras palatinas e faringianas ${ }^{7,13,17}$. Podem ainda ser utilizadas de maneira individual ou conjugada ${ }^{7}$.

Em se tratando especificamente da prótese ocular e oculopalpebral, a importância do uso das mesmas está intimamente ligara à manutenção de volume do osso orbital e, segundo Goel et al. ${ }^{11}$, pacientes enucleados que não foram reabilitados proteticamente apresentam colapso orbital.

Os materiais usados para confecção podem variar entre vidro (para as oculares), polimetilmetacrilato e silicone ${ }^{16}$. As de silicone, como no caso descrito, apresentam estabilidade química, resistência, durabilidade e facilidade de manipulação, entretanto, o material pode apresentar alterações de pigmentação com o passar do tempo e tal fato pode diminuir a satisfação do paciente com a prótese com o passar do tempo ${ }^{16,17}$. A adesão da prótese pode ser feita por meio de imãs, implantes, óculos ou, como no caso relatado, por meio de adesivos aplicados na interfase entre a pele e a peça ${ }^{13,17}$. Esses, entretanto podem causar reações alérgicas em alguns pacientes e colaboram para a alteração de cor nas bordas da peça devido a seu acúmulo nas margens ${ }^{13}$.

Os problemas relacionados ao desgaste das próteses maxilofaciais podem ser reduzidos e minimizados com 0 acompanhamento periódico do paciente, realizando ajustes e refazendo a peça, caso necessário; desse modo, é possível a manutenção de padrões funcionais e estéticos adequados ${ }^{17}$.

Deve-se deixar claro, no entanto, que tanto nesse caso como nos outros em que se opta por reabilitação facial protética, 0 preciosismo deve existir, mas a questão, nesse caso, vai além da perfeição, pois trata-se de proporcionar elevação da autoestima para um paciente mutilado pelo tratamento, que precisa se adaptar a sua nova condição e ter condições adequadas de sobrevida.

No caso relatado, viu-se uma jovem, mutilada por um tratamento invasivo, com vergonha da sua imagem, que pôde recuperar o aspecto de normalidade, evitado assim constrangimento social e psicológico. Desse modo, esse dispositivo representa um sucesso significativo no sentido de melhorar a autoestima e a qualidade de vida da paciente.

CONSIDERAÇÕES FINAIS

A reabilitação facial dos pacientes com 
tumores na região de cabeça e pescoço com próteses maxilofaciais é essencial no sentido de melhorar a autoestima do paciente assim como reinseri-lo na sociedade.

\section{REFERÊNCIAS}

1. Gray F, Ferlay J, Soerjomataram I, Siegel RL, Torre LA, Jemal A. Global cancer statistics 2018: GLOBOCAN estimates of incidence and mortality worldwide for 36 cancers in 185 countries. CA Cancer J Clin. 2018;68(6):394424.

2. Cohen N, Fedewa S, Chen AY. Epidemiology and Demographics of the Head and Neck Cancer Population. Oral Maxillofac Surg Clin North Am. 2018;30(4):381-95.

3. Lydiatt WM, Patel SG, O'Sullivan B, Brandwein MS, Ridge JA, Migliacci JC, et al. Head and neck cancers-major changes in the American Joint Committee on cancer eighth edition cancer staging manual. CA Cancer J Clin. 2017;67(2):122-37.

4. Bonanno A, Esmaeli B, Fingeret MC, Nelson D $\mathrm{V}$, Weber RS. Social challenges of cancer patients with orbitofacial disfigurement. Ophthal Plast Reconstr Surg. 2010;26(1):18-22.

5. Worrell E, Worrell L, Bisase B. Care of longterm survivors of head and neck cancer after treatment with oral or facial prostheses, or both. Br J Oral Maxillofac Surg. 2017;55(7):685-90.

6. Marur S, Forastiere AA. Head and neck squamous cell carcinoma: update on epidemiology, diagnosis, and treatment. Mayo Clin Proc. 2016;91(3):386-96.

7. Carvalho GD de, Souza LF de, Ferreira TO, Bento G, Haddad MF. Prótese bucomaxilofacial: a Odontologia além da boca. Arch Health Invest. 2019;8(6):322-28.

8. Gonçalves KV, Martins OBT, Neves M de S, Haddad MF. Reabilitação de paciente eviscerado por meio de prótese ocular: relato de caso. Arch Health Invest. 2018;7(8):329-33.

9. Hatamleh MM, Alnazzawi AA, Abbariki M, Alqudah N, Cook AE. Survey of ocular prosthetics rehabilitation in the United Kingdom, Part 2: Anophthalmic patients' satisfaction and acceptance. J Craniofac Surg. 2017;28(5): 1297-301.

10. Shankaran G, Deogade SC, Dhirawani R. Fabrication of a Cranial Prosthesis Combined with an Ocular Prosthesis Using Rapid Prototyping: A Case Report. J Dent (Tehran). 2016;13(1):68-72.

11. Goel GK, Jain D, Goel D, Juneja P. Rehabilitation after Surgical Treatment for Retinoblastoma: Ocular Prosthesis for a 6Month-Old Child. J Prosthodont. 2012;21(5):408-12.
12. Cardoso MSO, Araújo PGM, Cardoso AJO, Cardoso, S. M O, Morais LC. Implicações psicossociais em pacientes com perda do globo ocular. Rev Cir Traumatol. 2007;7(1):79-84.

13. Goulart DR, Sigua-Rodriguez EA, AlvarezPinzón N, Fernandes AUR, Queiroz E. Quality of life of patients with facial prosthesis. Rev Fac Odontol Univ Antioq. 2017;29:131-47.

14. Goulart DR, Queiroz E, Fernandes AÚR, De Oliveira LM. Aspectos psicossociais envolvidos na reabilitação de pacientes com cavidade anoftálmica: Implicações do uso de prótese ocular. Arq Bras Oftalmol. 2011;74(5):330-34.

15. Miracca RAA, Sobrinho J de A, Tanaka EMGT. Magnetos na Retenção de Prótese Conjugada Óculo- palpebral e Obturador Palatino. Rev Ibero-americana Prótese Clínica Lab. 2004;6(32):365-75.

16. Raizada K, Rani D. Ocular prosthesis. Contact Lens Anterior Eye. 2007;30(3):152-62.

17. Goiato MC, Pesqueira AA, Ramos da Silva C, Filho HG, Micheline dos Santos D. Patient satisfaction with maxillofacial prosthesis. Literature review. J Plast Reconstr Aesthetic Surg. 2009;62(2):175-80.

18. McBain HB, Ezra DG, Rose GE, Newman SP, Byron-Daniel J, Charlton R, et al. The psychosocial impact of living with an ocular prosthesis. Orbit. 2014;33(1):39-44.

\section{CONFLITO DE INTERESSES}

Os autores declaram não haver conflitos de interesse

\section{AUTOR PARA CORRESPONDÊNCIA}

\section{Ana Carolina dos Santos Menezes}

Praça da Cruz Vermelha, 23 - Centro, 20230-130 Rio de Janeiro- RJ, Brasil

Telefone (21)32071859

E-mail: ana.smenezes@gmail.com
Submetido em 04/06/2020 Aceito em 23/10/2020 